\title{
A New Diagnosis Method on Insulators with Measuring Contact Angles
}

\author{
Yan Liu ${ }^{1,3}$, Kai $\mathrm{Liu}^{3}$, Wei-liang $\mathrm{Tao}^{2 *}$, Ye $\mathrm{Zhu}^{3}$ \\ ${ }^{1}$ School of Electronic Information,Wuhan University, P.R. China \\ ${ }^{2}$ Laboratory of System Integrated and Faults Diagnostics, Wuhan University, P.R.China \\ ${ }^{3}$ State Grid Electric Power Research Institute, Wuhan, P.R.China \\ * Corresponding author’s Email: taowl2003@163.com
}

\begin{abstract}
Compared to other methods, insulator's contact angles detection can obtain more precise and more accurate hydrophobicity in parts of insulator, getting in-depth information like hydrophobicity of shed material, migration of the hydrophobicity after polluted insulator, attenuation and recovery characteristics of the insulation performance under certain conditions, all of which are its particular advantages. A new detection method of insulator's performance is proposed in this paper with measuring contact angles. With this method, the boundary curve of water drop on insulator's surface was extracted with image processing, then the polynomial fitting was deployed to fit the boundary curve, and contact angles of water drop were calculated by differentiate the polynomial deduced from the polynomial fitting. So the performance of the insulator can be detected and the process doesn't need manual intervention which makes the detect result accurate and objective. Experiments show that the root mean square error of the measurement is below $2 \%$.
\end{abstract}

Keywords: insulator; hydrophobicity; contact angle; image processing;

\section{Introduction}

Insulators are widely used in the power system. The safe running of insulators is one of the factors to determine the investment budget and safety level of the power system. To ensure the safe electricity transmission, it is necessary to seek an effective way to measure insulator's performance which determines whether there is a need to maintain or replace the insulators.

There are many methods to measure the insulator's performance at present, of which the visual detection $^{[1]}$ is widely adopted. However it not only request manipulators to observe insulators closely, but also need them to familiarize with various types of insulators' material, design and the frequent failure modes. Resistance detection detects the leakage current to get the insulation resistance which can reflect the performance of insulators. The method is simple and practical, but the information can be gained is limit. The effectiveness of the electric field detection methods ${ }^{[2-5]}$ has been verified through experiments mentioned by the literature $^{[6]}$. Meanwhile, it was found that the detection precision is related to the fault location and whether the insulator is inundated will also affect the results. Optical detection ${ }^{[7,8]}$ estimates insulator's discharge actions by observing the photons on insulator's sur- face. This method must avoid sunlight exposure, therefore requires the condition of night. Thermal detection ${ }^{[9-11]}$ estimates insulator's performance by the principles that insulator surface material degra- dation is associated with the quantity of heat emitting from the insulator in the electric field. The essence of this detection technology determines that it is sensitive to the influences from the wind, rain, sunshine and other environmental condition. Acous- tic detection detects the noise produced by discha- rges with 
microphone to estimates insulator's perfor- mance, and its feasibility has been proved by Lundgaard $^{[12]}$. The fault location and type of information can be obtained ${ }^{[13]}$ through analyzing the amplitude, frequency and phase of acoustic wave. But the effect of the detection is determined by the material of insulator.

Hydrophobicity detection technology is one of the main detection methods as regards the evaluation of insulator's performance. The traditional method measures the insulators' hydrophobicity through measuring the contact angle directly by photography ${ }^{[14]}$. However, this method can only be carried out in laboratory environment. The restriction is made up by STRI hydrophobicity classification method ${ }^{[15,16]}$. But it is dependent on the operators' subjective judgment, so the accuracy can not be high. The method mentioned in the Literature [17][18] estimates the average hydrophobicity using digital image processing. It is more objective and precise, but it bases on the hydrophobicity classification and can not get the insulator's partial hydrophobicity.

It is obvious that the existing detection methods are not satisfying and have some disadvantages. So experts nationally and internationally keep researching $^{[19]}$ in this field.

This paper presents a detection method of insulator's performance through measuring the hydrophobicity. The method takes advantage of image processing technology, such as extracting water droplets' image from the background, fitting the water droplets' contour using the orthogonal polynomial and measuring the contact angle, to estimate the insulator's hydrophobicity. Comparing with other hydrophobicity detection methods, this method can detect the insulators which are still running on the spot without manual interventions, and the measurement results are more objective and precise.

\section{Basic principle}

The definition of contact angle is shown in Figure 1. When the insulator's surface is horizontal, the contact angles of water droplet on both sides are the same, and is called static contact angle $\theta$. The dynamic contact angles $\theta_{a}$ (advancing angle) and $\theta_{r}$ (receding angle) can be defined when a droplet is on an inclined surface.

The model of contact angle detection method is based on image processing. Use drip device, drip appropriate water droplets on the insulator surface, then use CCD camera device to acquire images, image processing tools and curve fitting can be adopted to get contact angle.

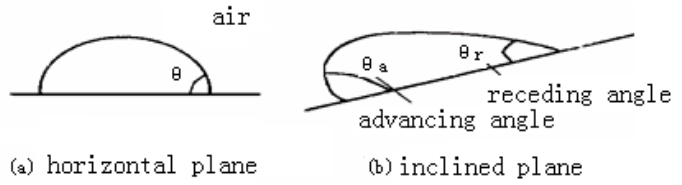

Figure 1 Diagram of contact angles

Obviously, in the same water droplet capacity and times, the larger the static contact angle, the smaller the interface that the water droplet contacting with the insulation material, the better the hydrophobicity. It is generally believed that, when $\theta>90^{\circ}$ insulator surface is hydrophobic. For inclined surface, by measuring the advancing angle and the receding angle, the following formula can be used to research hydrophobicity ${ }^{[20]}$.

$$
m g \sin (\alpha)=L \gamma\left(\cos \theta_{a}-\cos \theta_{r}\right)
$$

where $m$ is mass of water droplet, $g$ is acceleration of gravity, $\alpha$ is incline angle, $L$ is length of water droplet, $\gamma$ is liquid surface tension, and $\theta_{r}$ and $\theta_{a}$ are the largest advancing angle and receding angle of water droplet in inclined surface, respectively.

Based on the liquid surface tension insulator hydrophobic can be measured, the smaller the surface tension, the better the hydrophobic.

\section{Image processing}

Image processing includes image preprocessing, image segmentation, image feature extraction and curve fitting derivative about water droplet, etc.

\subsection{Water droplet image preprocessing}

1) Gray value transforming

For the operation in Gray Image is simpler than Colored Image, we often transfer Colored Image into Gray Image of 256 colors.

2) Image smoothing

Most pixels' gray value of an image is of little difference with the adjacent, existing large gray correlation degree, which leads the energy of the image to concentrate in the low-frequency region. Attenuating high-frequency components and enhancing low-frequency component can retain the main message of the image and filter high-frequency noise.

Image smoothing methods include: neighbor- 
hood average, median filter, Gaussian filter, frequency domain method and so on. Particularly, median filter is suitable for extracting water droplet contours from the image, which has the following advantages: (1) to preserve edge details while removing the impulse noise, salt and pepper noise at the same time. (2)to eliminate pulse effectively without affecting the step function and the slope function. (3) to avoid fuzziness on image detail resulted by the linear filter under certain conditions. (4) computing process is convenient without need of the statistical characteristics of images. Experimental results show that the average templates and Gaussian template both blur image, especially the average template, while the median template can preserver edge detail better, as shown in Figure 2. So in practice we choose median filtering method.

Detailed practice: to use a sliding window with odd point to slide on the image, to arrange the odd pixels with the pixel gray value, which are contained in the window, from small to large; then to replace the pixel gray value of the window centre with the middle gray value in the order, to move the window from left to right till reaching the border, then to move to the next row, from left to right again and go through the whole image in this way. After the median filter transformation, some pixel's gray value is equivalent to the middle value of the gray values in the neighborhood.

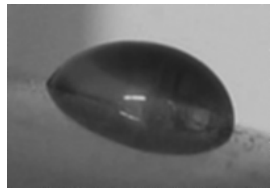

(a) original image

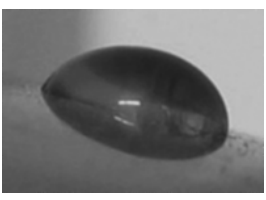

(c) Gaussian template filter

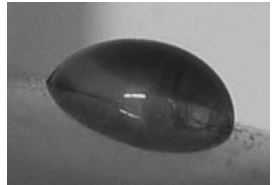

(b) average template filter

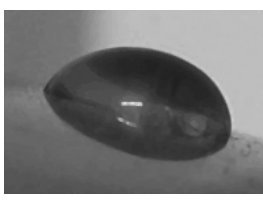

(d) median template filter
Figure 2 Images of three kinds of filtering templates

\section{3) Gray stretch}

Since water is translucent liquid, the contrast between water droplet image and its background is too little to distinguish. In order to highlight droplet and suppress the background, piecewise linear transformation is adopted here.

The gray value of the water droplet in the image is lower than the background, and the whole image has a low gray value. Therefore the high gray section is compressed and the low gray section is stretched.

To be simple, we can choose a gray value $M$, stretch the section where gray value is less than $\mathrm{M}$, and turn the section where gray value is more than $\mathrm{M}$ to the highest gray value totally. If $f(i, j)$ denotes pixel gray value of the original image, $g(i, j)$ denotes the gray value after gray transform, the linear transformation function would be:

$$
g(i, j)=\left\{\begin{array}{cc}
\left(M_{g} / M\right) f(i, j) & 0 \leq f(i, j) \leq M \\
M_{g} & M<f(i, j) \leq M_{g}
\end{array}\right.
$$

\section{4) Image Rotation}

According to the need of image processing that followed, we hope that the water droplet in the image is at the horizontal level on the whole in the picture. As the incline angle on the insulator surface is about $15^{\circ}$, the whole image should be rotated by $15^{\circ}$.

\subsection{Image segmentation}

\section{1) Edge detection}

The water droplet's transparency makes the boundary between water droplet and its background less obvious, particularly on the light side of the border. To detect and highlight the edge of the water droplet is the premise of extraction of water droplet contour accurately.

Edges mainly divide into step shape and roof shape. The water droplet image is step edge where the edge points' first-order derivative has extreme value. So detecting edge points can be achieved by calculating each pixel's gray gradient.

The digital image's first-order partial derivatives would approximately be:

$$
\left\{\begin{array}{l}
f_{x}^{\prime}=f(x+1, y)-f(x, y) \\
f_{y}^{\prime}=f(x, y+1)-f(x, y)
\end{array}\right.
$$

To simplify the calculation of gradient, the following approximate formula is used frequently:

$$
\operatorname{grad}(x, y)=\left|f_{x}^{\prime}\right|+\left|f_{y}^{\prime}\right|
$$

Generally, the gradient operator is sensitive in level or vertical direction on the edge, while Roberts across operator detects the gradient which cross along with the image coordinate axis $45^{\circ}$ and $135^{\circ}$. The Operator is:

$$
\begin{aligned}
& \operatorname{grad}(x, y)=\left|f_{x}^{\prime}\right|+\left|f_{y}^{\prime}\right|= \\
& |f(x, y)-f(x+1, y+1)|+|f(x+1, y)-f(x, y+1)|
\end{aligned}
$$


Other common operators include Prewitt operator, Sobel operator, and Laplace operator. As shown in Figure 3, it is indicated from the four edge detection experimental results that Laplace operator is more affected by noise, Prewitt and Sobel operator detection edge are wider and its accuracy is low, and Robert operator results turn up satisfactorily.

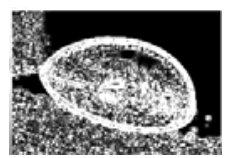

(a) Laplace operator

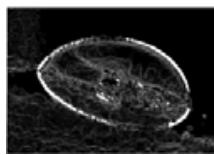

(c) Sobel operator

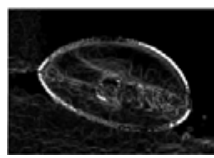

(b) Prewitt operator

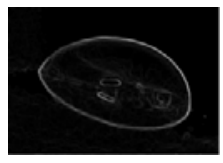

(d) Roberts operator

Figure 3 Images of four edge detecting algorithms

\section{2) Binary processing}

Binary processing makes the image pixel gray value either 0 or 255 with certain rules, so focused areas are apart from background completely. Among binary processing methods threshold segmentation is effective and easy to implement.

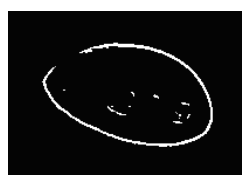

(a) OTSU method

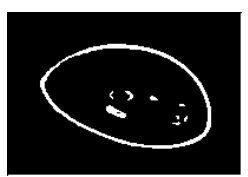

(b) Iterative method
Figure 4 Binarization images of droplet

Suppose one image is defined as following mapping: $f(x, y) \in G$, and $(x, y)$ is the space coordinates, $f(x, y)$ is the gray value on the position of $(x, y), \mathrm{G}$ is a positive integer set which denotes gray levels. Segment image with threshold $\mathrm{T}$, the result would be $f_{t}(x, y) \in\{0,255\}$, which is:

$$
f_{t}(x, y)=\left\{\begin{array}{cc}
255 & f(x, y) \geq T \\
0 & f(x, y)<T
\end{array}\right.
$$

Therefore, those pixels with gray value 255 correspond to the objects and the others correspond to the background.

The key of threshold segmentation is how to determine the optimal threshold value. As noises, such as facular noise, exist in water droplet image, segmentation results with fixed threshold would be extensively affected by the noise. OTSU method and iterative method are the main methods to achieve the optimal dynamic threshold value. As shown in
Figure 4, when the gray value between object and background is close, OTSU method will lose the object information, while iterative method results are better, so iterative method is adopted.

The approach can be described as follows.

Firstly, to calculate image gray value histogram, to select the median of the whole image gray range as an initial threshold $\mathrm{T}$ (suppose there are L grades of gray value in all). Then to use $\mathrm{T}$ to segment image, this will generate two sets of pixels. G1 set is made up of all pixels whose gray value is larger than or equal to T, and G2 set is made up of all the pixels whose grey value is less than the $\mathrm{T}$ value. Calculate the average gray value of all the pixels in G1 and G2:

$\mu_{1}=\sum_{m=0}^{T}\left(c_{m} \times m\right) / \sum_{m=0}^{T} c_{m}, \mu_{2}=\sum_{m=T+1}^{L-1}\left(c_{m} \times m\right) / \sum_{m=T+1}^{L-1} c_{m}$

Secondly, to calculate the new threshold $\mathrm{T}=0.5\left(\mu_{1}+\mu_{2}\right)$; then to calculate the new average gray value $\mu_{1}, \mu_{2}$ with this new threshold, and to repeat the steps above until the iteration value is equal to $\mathrm{T}$.

\subsection{Contour extraction}

1) Thinning of binary image

Thinning algorithm is stripping the border pixels from binary image repeatedly, until the width of border turns to a pixel. During thinning processing, the connectivity of the object must be maintained.

Thinning algorithm deployed in this paper is iterative algorithm for extract skeleton. It's a method to judge if a border point should be eliminated from the binary structural contour according to the relationship with adjacent points, to realize contour thinning.

Iterative algorithm is as follows: Firstly, it needs to detect the whole image, and to search the structural elements $\mathrm{p} 1$ to compute $\mathrm{n}(\mathrm{p} 1)$ and $\mathrm{s}(\mathrm{p} 1)$. Judging by the following four conditions:

Condition $1: 2 \leq n\left(p_{1}\right) \leq 6$ If $\mathrm{p} 1$ only has one neighborhood point, $\mathrm{p} 1$ is pixels string's end point, can't be deleted. If $\mathrm{p} 1$ has seven neighborhood points, to delete p1that will delete one region will lead a section split.

Condition 2: $\mathrm{s}(\mathrm{p} 1)=1$. If there have more than one adjacent point transition 0 to 1 , delete $\mathrm{p} 1$ will lead a section split.

Condition 3: $p_{2} \times p_{4} \times p_{6}=0$.

Condition 4: $p_{8} \times p_{4} \times p_{6}=0$.

If four conditions are met, as said p1 locates in the West, North or Southeast border of the objects as 
shown, P1 will be marked as "delete," after the points are all dealing with in images then delete reunification.

Then, repeat the above process to the processed image, but conditions 3 and conditions 4 are changed as follows:

Condition 3: $p_{2} \times p_{4} \times p_{8}=0$

Condition 4: $p_{2} \times p_{4} \times p_{8}=0$

Similarly, if four conditions are met at the same time, P1 will be marked as "delete," after the point is all dealing with in images then delete reunification. Then start a new iterative algorithm after completion until no pixel was marked as "delete."

\section{2) Contour tracking}

Besides getting the contour, some disordered noises still exist in the local area after thinning process. It is needed to extract water droplet borders more accurately. 8-neighborhood contour tracking algorithm can track and extract water droplet border effectively.

Eight direction codes on edge points are shown in Figure 5.

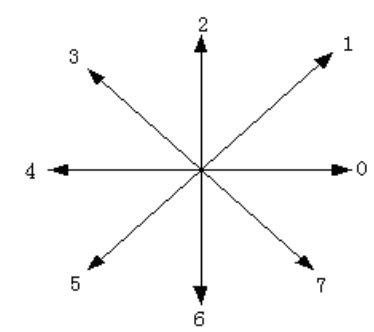

Figure 5 Eight direction codes on edge points

Suppose $(x, y)$ is a border point of the object, the next border point must lie in the 8-neighborhood area. So we can track outside border according to the 8-neighborhood information: Firstly, to locate a border point in the top left corner of the object area as a beginning for the search and then to search its 8-neighborhood for the next border point anti-clockwise, from top to bottom, from left to right, then to continue searching with the point as current starting point. This searching process will be repeated till we reach the first starting point back.

3) Vertical Scanning

For water is transparent, the aspect of water droplet towards the light side will be projected on the insulator and there is a "false border" in the image. So the lower part of the water droplet contour we get before is not the real border, but the projection on the insulator. We should take the most left droplet border point as the left point contacting the insulator, and the most right one as the right point contacting the insulator. Set the points which are above the line between two points as the gray background and only retain the true border which is on the top.

\subsection{Contact angle detection}

\section{1) Polynomial Fitting}

According to the definition of contact angle, to calculate it needs tangent slope at the contact point on the droplet border with the insulator. Therefore it is necessary fitting the discrete points in border curve, and working out the derivative of the endpoint in the curve function.

Generally, the least squares fitting algorithm which takes polynomial as basis function is adopted to march the curve fitting. But coefficient matrix of polynomial fitting (Gelanmu matrix) is a serious pathological matrix, which has an effect on computational stability.

Orthogonal polynomial fitting constructs a group of orthogonal polynomials as the basis function and makes their linear combination as curve equation. Its advantage is: the Gelanmu matrix is diagonal matrix, the result is stable and of less fitting distortion. So orthogonal polynomials are adopted as the basic function to fit.

2) Contact angle calculation

By curve fitting we have attained the fitting polynomial of original data points, from its derivative, the slopes of the endpoints can be calculated to obtain the advancing angle $\theta_{a}$ and the receding angle $\theta_{r}$.

\section{Experimental analyses}

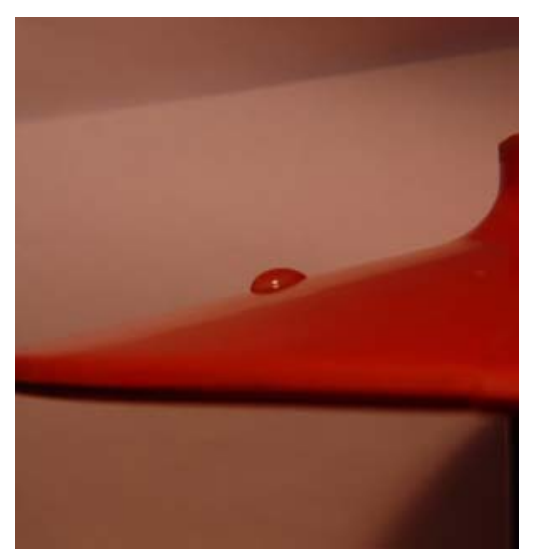

Figure 6 JPEG color image of water droplet 


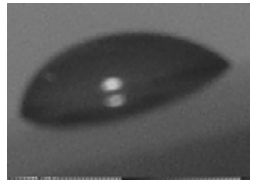

(a) gray transform

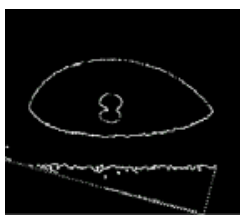

(f) binary processing

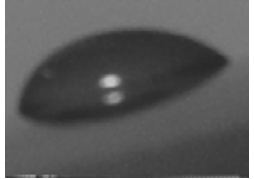

(b) median filter

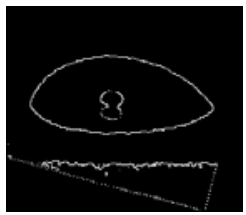

(g) thin processing

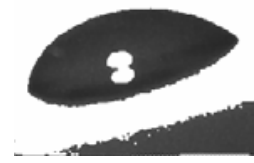

(c) gray stretch

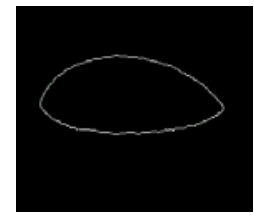

(h) contour tracking

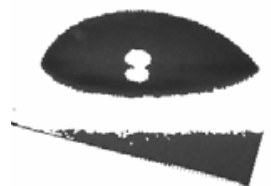

(d) Image rotation

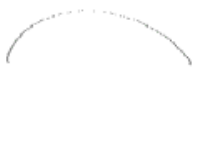

(i) vertical scanning

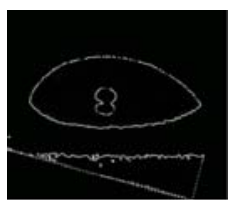

(e) Roberts edge detection

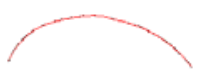

(j) polynomial fitting

Figure 7 The processing images of water droplet

In this paper Canon's digital camera "ixus60" is deployed to capture the water droplet image on insulator, whose resolution is $1600 \times 1200$ pixels and which is saved as the BMP format. Figure 6 shows the image.

Figure 7 shows images processing of the insulator contact angle detection. Since our attention is focused on the area near the endpoints, removing the mid-points which influence the fitting results and choosing a number of points near the endpoints to fit is likely to increase fitting accuracy.

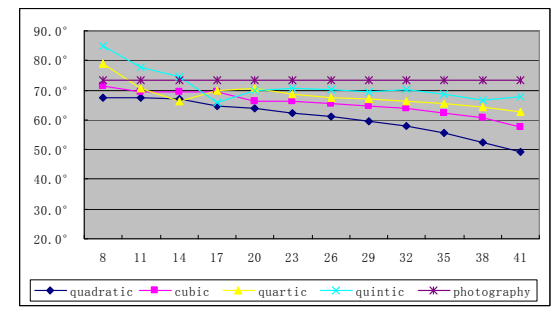

Figure 8 Precision analysis of advancing angle

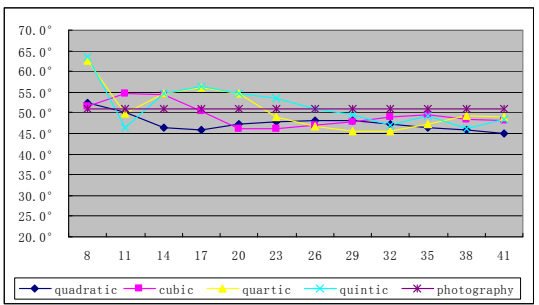

Figure 9 Precision analysis of receding angle

Choose different numbers of points near endpoints and polynomials degree with second, third, fourth or fifth to fit curve many times, the results are shown in Figure 8 and Figure 9.

The results show that when the polynomials degree is low, the fitting error is large, the contact angle has great fluctuation with fitting points number changing, and experiment reproducibility is poor. When polynomial degree is a higher number (more than four), the fitting effect is better. The calculated results are similar to photography measurement results. The less the fitting points are, the gentler the curve is, which illuminates that properly reduced sample points near the endpoints helps to enhance the accuracy. But when the number of points is less than 10, the results has a large fluctuation, which shows that there are not enough points, so the fitting accuracy is reduced and the detection accuracy is reduced either. All considered, we select 28 points near the endpoints to carry on five polynomial fitting.

Table 1 Result of contact angles measurement

\begin{tabular}{ccccc}
\hline Sequence & $\begin{array}{c}\text { Advancing } \\
\text { angle }\end{array}$ & $\begin{array}{c}\text { means } \\
\text { difference }\end{array}$ & $\begin{array}{c}\text { Receding } \\
\text { angle }\end{array}$ & $\begin{array}{c}\text { means } \\
\text { difference }\end{array}$ \\
\hline 1 & $74.0^{\circ}$ & $1.0^{\circ}$ & $54.6^{\circ}$ & $0.0^{\circ}$ \\
2 & $71.6^{\circ}$ & $1.4^{\circ}$ & $56.0^{\circ}$ & $1.4^{\circ}$ \\
3 & $73.8^{\circ}$ & $0.8^{\circ}$ & $53.6^{\circ}$ & $1.0^{\circ}$ \\
4 & $72.0^{\circ}$ & $1.0^{\circ}$ & $53.0^{\circ}$ & $1.6^{\circ}$ \\
5 & $73.5^{\circ}$ & $0.5^{\circ}$ & $55.8^{\circ}$ & $1.2^{\circ}$ \\
\hline Average & $73.0^{\circ}$ & $0.94^{\circ}$ & $54.6^{\circ}$ & $1.04^{\circ}$ \\
\hline
\end{tabular}

Using this whole process to measure the same insulator many times, we can get the results as shown in Table 1 , from which it is proved that measurement variance is less than $2 \%$. Thus the method is accurate, effective and has a certain 
perspective of practice.

\section{Conclusion}

In this paper, the detection method of hydrophobicity on insulator surface based on image processing could detect the contact angle with digital image processing techniques automatically. But further work should be done to make this method realized, such as how the image are gotten and how the image processing are taken in an embedded system. In addition, how the partial hydrophobicity is synthesized to evaluate the performance of an insulator and how the estimation of contact angles are affected when many water droplets with different sizes are present should be considered. Now this method provides the theory basic for the development of the insulator hydrophobicity online detection equipment.

\section{References}

[1] Cigré Working Group 22.03 (convener C. de Tourreil), "Worldwide service experience with HV composite insulators”, In: Electra, Vol.191, pp.2743, 2000.

[2] SHEN Xiaojun, JIANG Xiuchen, ZENG Yi, et al, "Harmonic electric field method for live detecting DC insulator", In: Automation of Electric Power Systems, Vol.29(15), pp.63-67, 2005.

[3] Zhao Hanbiao, Lin Hui, Xie Lili, et al, “On-line monitoring system on measuring leakage current near HV side for electrical transmission line insulators”, In: Automation of Electric Power Systems, Vol. 28(22), pp. 78-82, 2004.

[4] YANG Fan, LI Jiaxiang, YAO Degui, et al, “Charge simulation method base insulator non-touching electric field mapping method”, In: Automation of Electric Power Systems, Vol.28(15), pp.95-99, 2004.

[5] GAO Qiang, ZENG Chang’an, LI Heming, “A study of contamination detecting of insulators using the complex dielectric constant”, In: Automation of Electric Power Systems, Vol.31(18), pp.72-76, 2007.

[6] G. H. Vaillancourt, S. Carignan, and C. Jean, "Experience with the detection of faulty composite insulators on high-voltage power lines by the electric field measurement method", In: IEEE Trans. On Power Delivery, Vol.13 (2), pp.661-666, 1998.

[7] C. de Tourreil, "Status of inspection techniques to assess the condition of non-ceramic insulators in service”, In: Proc. Insulator 2000 World Congress, pp. 222-230, Barcelona, Spain, 1999.

[8] HE Wei, CHEN Tao, LIU Xiaoming, et al, “On-line monitoring system of faulty insulator based on non-touching UV pulse method”, In: Automation of Electric Power Systems, Vol.30(10), pp.69-74, 2006.
[9] E. Spangenberg and G. Riquel, "In service diagnostic of composite insulators EDF's test results", In: Proc. 10th Int. Symp. on HV Eng., Vol.4, pp.139-142, Montréal, Québec, Canada, 1997.

[10] HE Hongyin, YAO Jiangang, JIANG Zhenglong, et al, "Infrared thermal image detecting of high voltage insulator contamination grades based on support vector machine”. In: Automation of Electric Power Systems, Vol.29(24), pp.70-74, 2005.

[11] HE Hongying, YAO Jiangang, LUO Diansheng, et al,"Characteristic extraction method of contaminated insulator infrared image based on K-L transform”. In: Automation of Electric Power Systems, Vol.30(17), pp.76-80, 2006.

[12] L. E. Lundgaard and W. Hansen, "Acoustic method for quality control and in-service periodic monitoring of medium voltage cable terminations", In: Proc. 1998 IEEE Int. Symp. on Electrical Insulation, Vol.1, Arlington, Virgina, USA, pp.130-133, 1998.

[13] A. J. McGrail, D. W. Auckland, and B. R. Varlow, "Detecting and classifying flaws within insulating materials using ultrasound”, In: Proc. 1994 IEEE Int. Symp. on Electrical Insulation, pp.22-24, Pittsburgh, PA, USA, 1994.

[14] Y. C. Chen, C. R. Li, X. Liang, and S. Wang, "The influence of water and pollution on diagnosing defective composite insulator by electric field mapping”, In: Proc. 11th Int. Symp. on HV Eng., Vol.4, London, UK, pp.345-348, 1999.

[15] “STRI Hydrophobicity Classification Guide”. In: Swedish Transmission Research Institute, 1992.

[16] A. Eriksson and D. Wikström, "Influence of the hydrophobicity of outdoor insulators on the flashover voltage”, In: Proc. Nordic Insulation Symp. (Nord-IS '94), pp.39-51, Vaasa, Finland, 1994.

[17] T. Tokoro, Y. Omoto, and M. Kosaki, "Image analysis of hydrophobicity of polymer insulators using PVM", In: Proc. IEEE Conf. on Electrical Insulation and Dielectric Phenomena, pp.581-584, Kitchener, Ont., Canada, 2001.

[18] PENG Kexue, WANG Quande, WANG Xianpei, "Spray image analysis based measurement of hydrophobic of insulator surfaces”. In: Insulating Materials, Vol.1, pp.47-51, 2005.

[19] Gubanski, S.M. Dernfalk, A. Andersson, and J. Hillborg, H, "Diagnostic Methods for Outdoor Polymeric Insulators”, In: Dielectrics and Electrical Insulation, IEEE Transactions on, Vol.14(5), pp.1065-1080, 2007.

[20] ZHU Hong, MA Tiehua. "Utilization computer software dealing with and calculating the contact angle of solid-liquid gas interface”. In: Metrology and Measurement Technique, pp.39-41, 2004. 